\title{
UNDERSTANDING THE DYNAMIC MOMENTUM APERTURE OF THE ADVANCED LIGHT SOURCE*
}

\author{
C. Steier, D. Robin, Y. Wu, LBNL, Berkeley, CA94720, U.S.A.; \\ W. Decking, DESY, Hamburg, Germany; J. Laskar, L. Nadolski, IMC-CNRS, Paris, France
}

\begin{abstract}
The lifetime of a light source with small emittance like the Advanced Light Source (ALS) is usually limited by the momentum acceptance of the ring. Large momentum acceptances are reached by providing enough RF voltage and by avoiding a degradation of the dynamic momentum aperture. At the ALS the size of the momentum acceptance depends strongly on the transverse dynamics. It is very sensitive to machine conditions such as the tunes and chromaticities since depending on those conditions the Touschek scattered particles explore different resonance regions in the phase space. In this paper we show that by using a single-turn 'pinger' magnet together with turn-byturn beam position monitors (BPM) one can identify the cause of a reduction in momentum acceptance and take steps to improve the acceptance.
\end{abstract}

\section{INTRODUCTION}

The ALS is a third generation synchrotron light source located at Lawrence Berkeley National Laboratory [1]. Like all other light sources, the beam lifetime is one of the most crucial performance parameters for the users, since it has a large impact on the stability of the experiments and on the amount of integrated brightness which is delivered to the experiments. Similar to all other third generation light sources the lifetime is dominated by the Touschek effect. The Touschek lifetime depends strongly on the momentum acceptance of the machine.

Several effects contribute to the overall momentum acceptance (and therefore the Touschek lifetime): The rfbucket height, the physical apertures and in many cases the dominating effect: the dynamic aperture $[2,3]$.

To achieve small equilibrium emittances in third generation light sources, strongly focusing quadrupoles are used. This requires strong sextupoles to compensate for the chromatic aberrations in order to damp head-tail modes. The sextupole fields generate geometric and nonlinear chromatic aberrations which can excite resonances. Those resonances can cause the particle motion to become unstable or chaotic. In the ALS, low order resonances are suppressed by the 12 -fold periodicity of the ring.

\section{RF AMPLITUDE SCANS}

Scanning the rf-bucket height while measuring the beam lifetime can be used to quantify the effects determining the

\footnotetext{
* This work was supported by the U.S. Department of Energy, under Contract No. DE-AC03-76SF00098.
}

momentum acceptance. Because of the energy distribution of Touschek scattered particles one expects the lifetime to be a quadratic (to cubic) function of the RF-bucket height, as long as there are no dynamic effects limiting the acceptance. The turnover point, at which a deviation from the quadratic behaviour occurs, provides information about the nonlinear dynamics effects limiting the dynamic acceptance. Fig. 1 shows three scans of the RF-voltage for different linear chromaticities of the ALS.

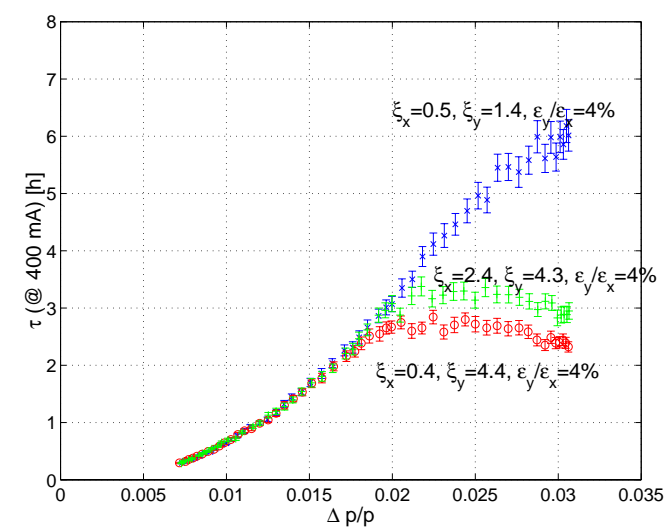

Figure 1: Scan of the RF amplitude and therefore the longitudinal bucket height for three different sets of horizontal and vertical chromaticities $(1.5 \mathrm{GeV})$.

As one can clearly see, the dynamic momentum acceptance and therefore the maximum achievable lifetime is very sensitive to the linear chromaticities. It is best for the nominal chromaticities of $\xi_{x}=0.5$ and $\xi_{y}=1.4$, which are used at the ALS in multibunch operation (blue 'x' symbols), and significantly worse (red 'o' symbols) for a vertical chromaticity increased by 3 units. Unfortunately, instabilities require us to increase the vertical chromaticity in two-bunch operation (up to $35 \mathrm{~mA} /$ bunch), to provide enough Landau damping. Therefore it is of interest to understand the effect causing this difference (which at first is surprisingly large, considering that the strength of sextupoles is only different by a few percent). The green '+' symbols indicate a case where based on the studies described later a lifetime improvement of about $25 \%$ was achieved for the high chromaticity case.

\section{LOSS MECHANISM}

To study and understand the effects limiting the dynamic momentum acceptance, some methods of Frequency Map Analysis [4] were modified to study the evolution of offenergy particle trajectories. Frequency Map Analysis is a very effective tool to visualize the global dynamics for sys- 
tems of 3 and more degrees of freedom. Originally it had been used at the ALS to study the dynamics of particles with nominal energy.

By simultaneously recording beam currents and therefore loss rates, the on-energy studies had shown, that certain resonance intersections can lead to diffusion and subsequent particle loss [5]. Due to radiation damping, all particles launched to amplitudes above such a loss region eventually have to damp back through the region, yielding some probability for particle loss. To verify this model, tracking studies were carried out, confirming that indeed particles damping through a loss region, might experience large diffusion and get lost (or as in the case of Fig. 2, get close to or outside of a physical aperture, which in the ALS is about $4 \mathrm{~mm}$ vertically).
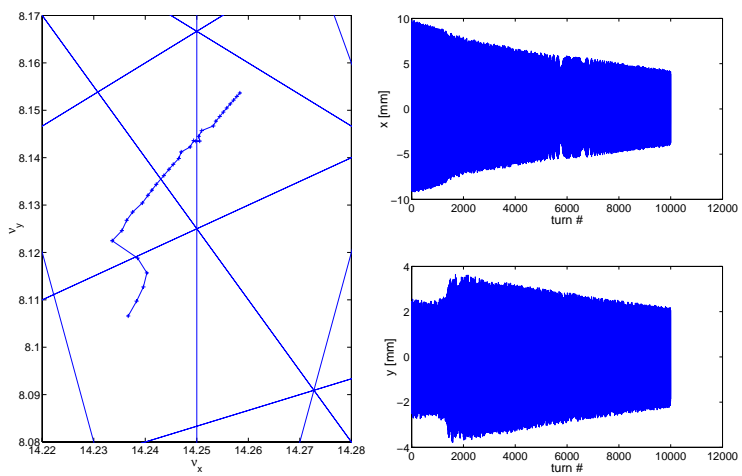

Figure 2: Single particle tracking with synchrotron radiation. Left: frequency space (tune calculated every 200 turns), right: configuration space.

\section{PINGER MEASUREMENTS}

To study the momentum acceptance in detail, two singleturn "pinger" magnets together with turn-by-turn beam position monitors (BPM) were used. To collect one data set, the RF-frequency was set to different values, corresponding to different beam energies. For each setting of the RFfrequency, the beam was then kicked with increasing horizontal amplitude (and a small vertical amplitude) while the beam oscillations and the fraction of beam loss were recorded, until the beam was completely lost.

The data sets for the nominal and high vertical chromaticity cases are shown in Fig. 3 and 4, respectively. The horizontal axis is the relative energy deviation, the vertical axis the kick amplitude in mm. The thickness of the dots indicates the relative size of the beam loss. The solid line shows to which horizontal amplitude a particle is launched, if it receives a given change in energy due to Touschek scattering in a region with dispersion at the ALS (i.e. the arcs). The intersection of these lines with the loss regions (areas with thick dots) corresponds roughly to the dynamic momentum acceptance.

The agreement between the estimated dynamic momentum acceptance from those measurements and the direct measurement using the RF-amplitude scans is very good. In addition one clearly sees completely different structures
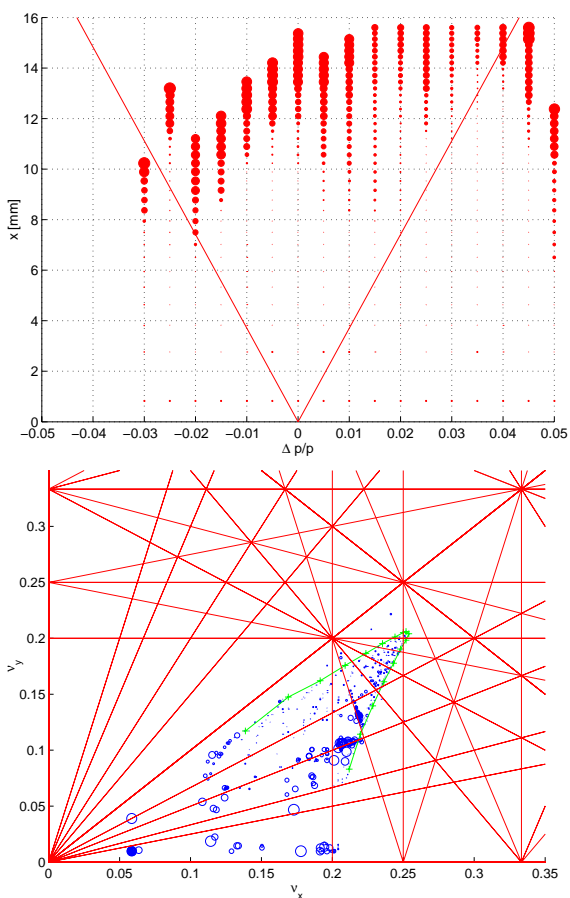

Figure 3: Measurement data showing the relative beam loss in configuration space (upper plot, $\beta_{x}=12 \mathrm{~m}$ ) and in tune space (lower plot) for the low chromaticity case. The dot thickness corresponds to the amount of normalized beam loss. The solid green line marks the tune shift with energy for particles with very small oscillation amplitudes.

in the shape of the loss regions in the two cases. Using the beam oscillation data, which was recorded at the same time as additional information, one can identify which resonances in tune space cause those loss regions. The analysis showed (compare Fig. 3 and 4) that the large difference in lifetime is not caused by a large difference in strength of resonances, but by the fact that different linear chromaticities cause the particles to sample completely different regions in phase space. Because of that, they experience completely different sets of resonances.

The large loss region at positive energy deviations in Fig. 4 turned out to be caused by the coupling resonance, which is intentionally excited at the ALS to increase/control the vertical beam size in order to achieve acceptable lifetimes. In the case with low vertical chromaticity on the other hand, the tunes do not cross the coupling resonance. Based on this it was predicted, that raising the horizontal chromaticity simultaneously should help to increase the momentum acceptance in the high vertical chromaticity case by shifting the crossing point with the coupling resonance to larger energy deviations. The measurements indeed showed that there was an improvement of about $25 \%$ in lifetime which immediately benefits the two-bunch operation of the ALS.

\section{COMPARISON WITH SIMULATIONS}

Simulations based on tracking with the calibrated model of the ALS [5] are in very good agreement with the mea- 


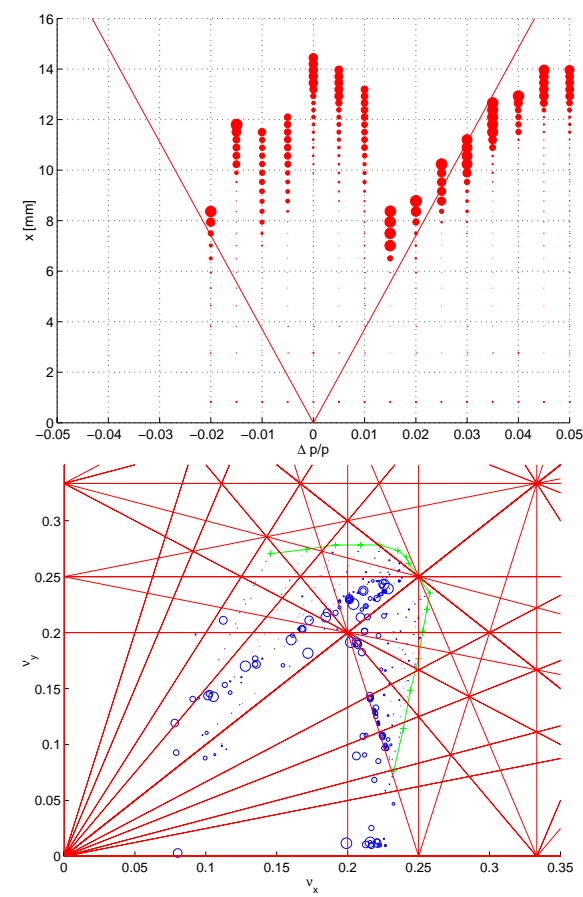

Figure 4: Measurement data for the case with higher vertical chromaticity. Significantly different areas of the tune space are sampled compared to the low chromaticity case.

surements (see Fig. 5, where the high vertical chromaticity case is shown). The color code in the plots indicates the diffusion rate (as defined by the change in betatron tune in one turn) on a logarithmic scale. Blue areas indicate low diffusion, red areas high diffusion and areas without color indicate unstable regions.

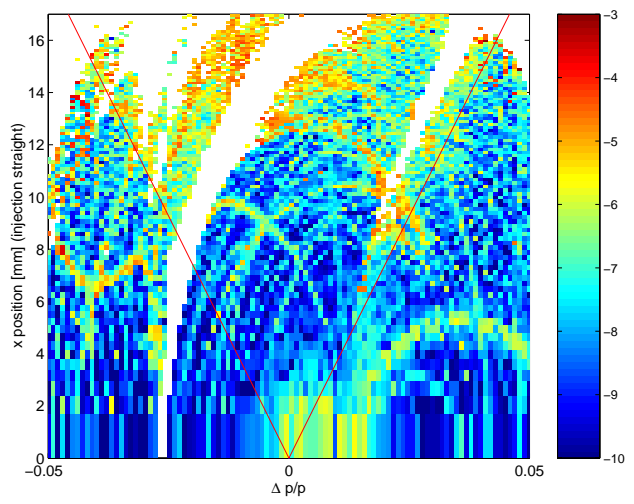

Figure 5: Simulation of a frequency map with the energy offset and the horizontal oscillation amplitudes as the variables spanning the configuration space. The color code indicates the diffusion rate on a logarithmic scale.

\section{APPLICATION TO THE ALS SUPERBEND UPGRADE}

To satisfy the demand for more high energy, high brightness beamlines at the ALS, a project is underway to replace three regular bend magnets with three $5.75 \mathrm{~T}$ superconducting magnets (Superbends) [6] in August of 2001. This will impact the symmetry of the lattice functions and therefore the single particle dynamics. Extensive simulation studies have been carried out to ensure that the impact on the machine performance is small. To study the impact experimentally before the actual installation of the Superbends, three pairs of additional quadrupoles in the Superbend sectors were used to simulate the symmetry breaking as closely as possible. Using frequency analysis to analyze the experimental dynamic momentum acceptance studies proved very valuable. It allowed to immediately understand initial problems and to verify that the impact of the Superbends indeed will be minimal, as long as the tunes and chromaticities are kept close to their nominal values.

\section{SUMMARY}

Measurements have demonstrated that the dynamic momentum acceptance in the ALS is the dominant effect determining the Touschek lifetime. Employing the method of frequency analysis provides a very powerful model independent diagnostic tool to visualize the global dynamics of the system, understand limitations and suggest improvement strategies. Even with the limitation of only two available sextupole families in the ALS, a significant improvement of the lifetime in two bunch operation was realized. For newer light sources with very elaborate sextupole configurations, this method can help to systematically optimize the momentum acceptance.

The agreement between measured data and simulation results which are based on calibrated machine models is good, providing both confidence in the model and the possibility to accurately predict the performance of upgrades to the ALS or the performance of new machines.

To make the method more usable as a tool for online optimization of the accelerator, we plan to reduce the measurement time significantly by upgrading the control system interface of BPMs and the pinger magnets. Moreover we plan to study a case nearly identical to Touschek scattering by applying transverse and longitudinal kicks simultaneously.

\section{REFERENCES}

[1] 1-2 GeV Synchrotron Radiation Source Conceptual Design Report, LBNL publication PUB-5172 Rev. (1986)

[2] W. Decking and D. Robin, "Momentum Aperture of the Advanced Light Source", AIP conf. proc. 468 (1998), p. 119

[3] W. Decking and D. Robin, "Dynamic Aperture Measurements at the Advanced Light Source", Proceedings of PAC 1998, New York, USA

[4] D. Robin, C. Steier, J. Laskar, L. Nadolski, "Global Dynamics of the Advanced Light Source Revealed through Experimental Frequency Map Analysis", Phys. Rev. Lett. 85, 3, (July 2000), pp. 558-561

[5] C. Steier et al., "Lattice Model Calibration and Frequency Map Measurements at the ALS”, Proceedings of EPAC 2000, p. 1077, Vienna, Austria

[6] D. Robin et al., "Superbend Project at the Advanced Light Source", these proceedings 